\title{
Performance Comparison of Modulation Schemes and Adaptive Modulation Scheme with CQI Feedback
}

\author{
Authors: Pratik Matkar ${ }^{1}$; Prasham Jain ${ }^{2}$ \\ Department of Electronics and Communication Engineering, Medicaps Institute of Science and \\ Technology, Indore (M.P.) $)^{1,2}$
}

E-mail:pratikmatkar1@gmail.com ${ }^{1}$;prasham55@gmail.com ${ }^{2}$

\section{DOI <10.26821/IJSHRE.6.6.2018.6605>}

\begin{abstract}
This paper includes a little bit addition to lot of work done to improve the Spectral efficiency of mobile communication system in LTE. The improvement in spectral efficiency is done by increasing data rate through adapting higher order modulation schemes or higher dimension MIMO techniques. But in noisy channel condition, such selection may increase the probability of error, thus leads to lesser throughput. In this Paper, we have optimized the performance of the MIMO trans-receiver by comparing its performance in case of various modulation schemes and Adaptive modulation schemes using the CQI feedback. A comparison graph has been drawn to show the performance comparison on the basis of RMS Error vs SNR $d B$ curve and thus comparing the data rate and error rate for various SNR values for different schemes.
\end{abstract}

Keywords: Spectral Efficiency, Modulation, Channel Quality Indicator (CQI), MIMO, Adaptive Feedback.

\section{INTRODUCTION}

Spectral efficiency is an important parameter to evaluate the performance of mobile communication system. The LTE standard has specific requirements in terms of overall spectral efficiency relative to $3 \mathrm{G}$ (third-generation) standards. For a given bandwidth allocation, you can increase the spectral efficiency by augmenting the data rate through the use of higherorder modulation or higher-dimension MIMO techniques; in noisy channel conditions, however, such a selection may increase the probability of error and thus have a detrimental effect on the effective throughput.

In order to achieve the desired spectral efficiencies consistently, the $3 \mathrm{G}$ and $4 \mathrm{G}$ standards, including the LTE, employ techniques that dynamically change system parameters based on channel conditions. These techniques are generally known as channel-aware scheduling or link adaptations. The basic idea of link adaptation is to adapt certain transmission parameters to varying channel conditions as they are monitored and measured by the system. Typical system parameters that are dynamically adapted include the system bandwidth, MIMO transmission modes, the number of transmission layers, the precoding matrix, Modulation and Coding Schemes (MCSs), and transmission power. With proper selection of these system parameters, we can exploit bandwidth resources more effectively instead of using a fixed parameter set that provides the best performance only in a worst-case channel condition. [1]

Here we will review various measurement made in the mobile receiver in order to ascertain the channel conditions as a function of time. These include Channel Quality Indicator (CQI), Precoder Matrix Indicator (PMI), and Rank Indicator (RI) measurements of which we will take up CQI only. Then we will discuss adaptation techniques that respond to channel measurements and change various system parameters to maintain a given measure of quality. These include adaptations of MCS, adaptive pre-coding in closed-loop spatial multiplexing modes, and adaptive MIMO based on rank estimation. This includes adaptations of MCS only. 
Volume 6 Issue 6 June, 2018

\section{SYSTEM MODEL}

Link adaptation is all about adapting to the channel conditions and changing system parameters based on actual channel quality. The LTE standard enables link adaptations that can help us make use of the spectrum more efficiently. The cost associated with this adaptation is the additional computational complexity involved in implementing link-aware schedulers.

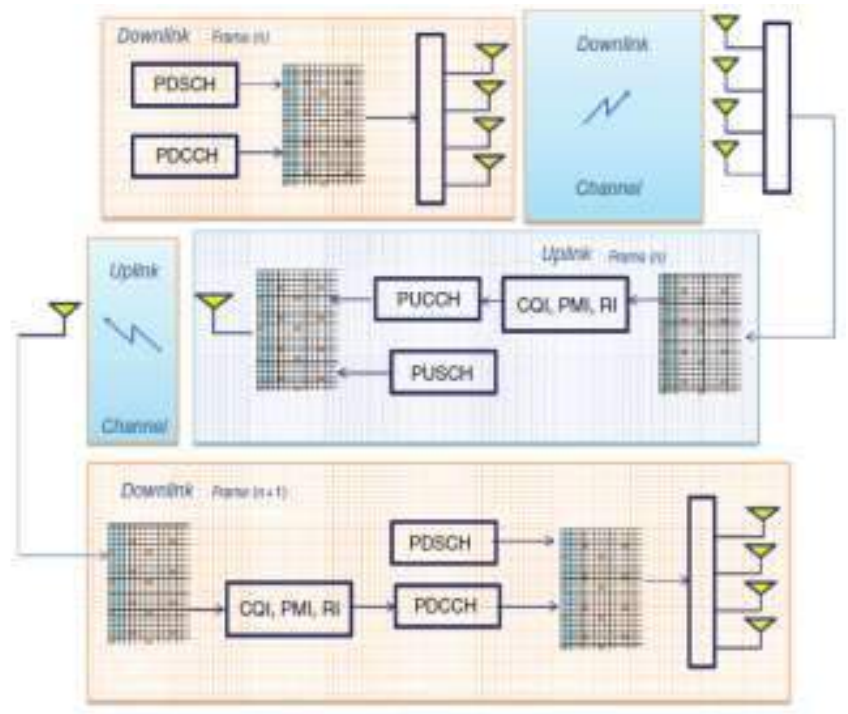

Fig 1: Sequence of downlink and uplink operations involved in link adaptations. [3]

The series of operations performed in a typical link adaptation scenario can be summarized as follows.

1. At subframe (n) (refer figure 1), the downlink transmitter forms the resource grid from the user data (PDSCH, Physical Downlink Shared Channel) and the Downlink Control Information, DCI (the PDCCH). The DCI contains the scheduling assignments that help the mobile receiver correctly decode the subframe information. The information contained in the PDCCH includes the MCSs, the precoder matrix, rank information, and the MIMO mode used. [3]

2. The mobile receiver can then perform the critical step of channel condition measurement as part of the process of decoding the received resource grid. In this process, it estimates the received channel matrix and performs various channel quality measurements. These measurements include the CQI, the PMI, and the RI. [3]

3. As part of uplink transmission, the mobile (UE) transmitter may embed the channel quality measures within the $\mathrm{PUCCH}$ and transmit to the base station (eNodeB) as a closed-loop feedback mechanism. [3]

4. The base station (eNodeB) receiver can then decode the PUCCH information to obtain channel measurements. Having this information available enables the system scheduler to decide whether or not to adapt various system parameters in the next frame as a result of feedback received from downlink channel quality. [3]

5. At the base station (eNodeB) in the downlink transmitter operations for the next subframe $(n+1)$, the scheduling decisions based on channel conditions are encoded into the PDCCH information and transmitted to the mobile. These include the new MCSs, precoder matrix, rank information, and MIMO mode that are now adapted based on the actual channel quality in the last subframe $(n)$. This full feedback process is then repeated for each subframe. [3]

\section{LINK ADAPTATION IN LTE}

To enable dynamic changes to MCSs and for proper operation of MIMO schemes, the LTE standard provides mechanisms that enable information regarding the channel characteristics to be measured by the mobile unit (UE). This information is then fed back to the base station (eNodeB) to help with scheduling and link adaptation.

At the mobile receiver, three types of channel-state report are generated and transmitted to the base station: 1. The CQI, a measure of downlink radio channel quality that specifies the best modulation constellation and coding rate to match the link quality.

2. The PMI, a measure that indicates the best set of precoding matrices for use in closed-loop single- and multi-user spatial multiplexing modes of the LTE standard.

3. The RI, which signals the number of useful transmission layers that can be used by the transmitter in spatial multiplexing modes.

\subsection{Channel Quality Estimation}

The CQI report gives a measure of the mobile radio channel quality. It provides a recommendation concerning the best MCS for the communication channel. The value of this measure is computed such that the transport block error rate using this recommendation will not exceed $10 \%$. The higher the value of the CQI measure, the higher the modulation order and the higher the coding rate. There are two 
Volume 6 Issue 6 June, 2018

types of CQI report, based on their granularity: a wideband CQI report assigns a single MCS value for the whole system bandwidth, while a sub-band CQI report assigns multiple MCS values to different contiguous resource blocks.

Most of these techniques select the best MCS as a function of the post-detection SINR (Signal-toInterference and Noise Ratio) measure. This measure is selected such that the Packet Error Rate (PER) experienced in the transmission is less than a given target. This in turn allows the system to avoid frequent retransmissions. The best MCS recommendation can ultimately be selected by quantizing the SINR value using a codebook lookup table. [3]

\subsection{CQI Estimation}

The two MATLAB functions in this section implement the channel quality estimation (CQI) measure based on the SINR of the MIMO receiver output and the transmitted signal. The CQI estimation is performed in two steps:

1. SINR estimation: The SINR measure is computed as a function of the decoded bits in the receiver and the MIMO receiver output

2. Spectral efficiency lookup: The computed SINR values are mapped to a spectral efficiency measure defined as the product of the number of modulated bits per symbol and the coding rate. For each SINR measure, distinct modulation schemes are found through a table lookup.

We implement a transformation that maps the SINR estimate to the proposed modulation scheme and the coding rate using a 4 bit (16-interval) scalar quantizer, we first map the SINR values to a CQI index, Then perform the mapping operation. The threshold values correspond to the boundary points of the scalar quantizer. Since the quantizer is defined as unbounded (input values have a range from -inf to +inf), only 15 threshold values are needed to subdivide the real axis into 16 regions represented by four CQI bits. The threshold values mapping the SINR values to the spectral efficiency are based on a simple lookup table.

In order to compute the modulation scheme and the coding rate outputs, we perform a table lookup operation with the CQI index. For the first seven values of the CQI index (indices 0-6), we map to a QPSK (Quadrature Phase Shift Keying) modulation with a modulation rate of 2 bits per symbol. The next three CQI indices (7, 8, and 9) are mapped to the 16QAM (Quadrature Amplitude Modulation) modulator with a modulation rate of 4 bits per symbol. Finally, the last six CQI indices (10-15) are mapped to 64QAM with a 6-bitsper- symbol modulation rate. Technically, the CQI index 0 signals an out-of-range message and does not participate in modulation mapping.

Table 1. Lookup table for mapping SINR estimate to modulation scheme. [3]

\begin{tabular}{|c|c|}
\hline CQI INDEX & MODULATION \\
\hline $1-6$ & QPSK \\
\hline $7-9$ & 16 QAM \\
\hline $10-15$ & 64 QAM \\
\hline
\end{tabular}

\subsection{Adaptive Modulation}

In this section we take advantage of the CQI channelstate report to adaptively change the modulation scheme of the transceiver in successive subframes. We implement a wideband modulation selection, in which in any given subframe all resource blocks will have the same modulation scheme and the change occurs between subframes. To understand the design tradeoffs, we need to compare adaptive modulation with alternative implementations. We feature two algorithms that apply different adaptation scenarios: (i) baseline (with no adaptation), and (ii) adaptation by exploitation of the CQI channel measurements.

\subsection{Parameters}

The selection of parameters is based on the simulation from LTE system toolbox in MATLAB.

Table2. Parameters for Simulation

\begin{tabular}{|l|l|}
\hline Transmission Mode & Spatial multiplexing \\
\hline Transmit antenna & 1 or 2 \\
\hline Receive antenna & 1 or 2 \\
\hline Modulation Type & QPSK, 16QAM, \\
\hline Code Rate & 0.3333 \\
\hline Channel model & Frequency selective \\
\hline Doppler & 120 \\
\hline Equalization & MMSE \\
\hline SNR dB & $1: 20$ \\
\hline
\end{tabular}


Volume 6 Issue 6 June, 2018

\section{RESULT AND ANALYSIS}

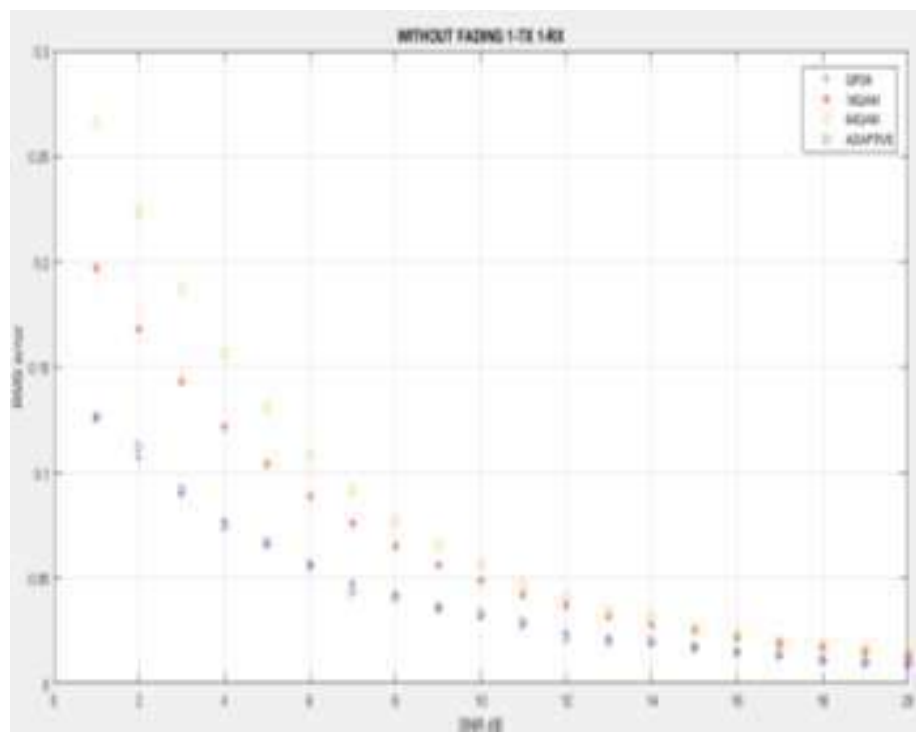

Fig 2: Plot of RMS error vs SNR for without fading 1-Tx 1-Rx

The plot (fig. 2) has been shown for static MIMO channel model (without fading) and SU-MIMO that is one transmit and receive antennas which shows that with the increasing SNR values the data rate increases with increasing error for higher modulation schemes. The statistical values of error shows that adaptive modulation gives optimum results for SNR. (Refer fig. 2)

\section{WTH FW}

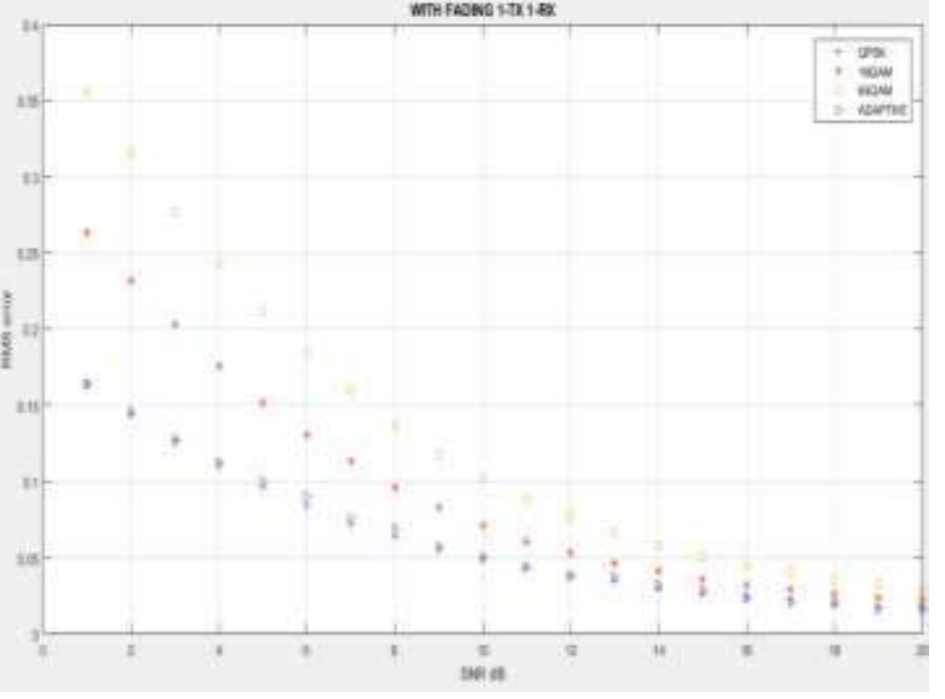

Fig 3: Plot of RMS error vs SNR for with fading 1Tx 1-Rx

The plot (fig. 3) has been shown for dynamic MIMO channel model (with fading) and SU-MIMO that is one transmit and receive antennas which shows that with the increasing SNR values the data rate increases with increasing error for higher modulation schemes. Here due to fading we can see that the channel becomes more noisy and hence error increases for all modulation schemes. The statistical values as well as plot of error shows that adaptive modulation gives optimum results for given SNR. (Refer fig. 3)

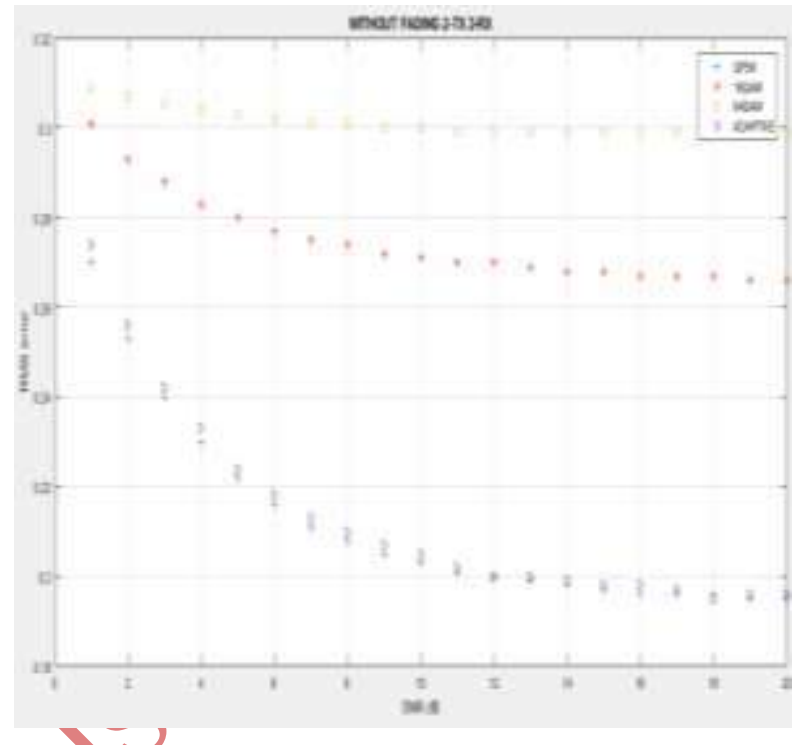

Fig 4: Plot of RMS error vs SNR for without fading 2-Tx 2-Rx

The plot (fig. 4) has been shown for static MIMO channel model (without fading) and MU-MIMO that is two transmit and receive antennas which shows that with the increasing SNR values the data rate increases with increasing error for higher modulation schemes. Here due to increased no. of transmit and receive antenna interference increases and hence error increases for all modulation schemes. The statistical values as well as plot of error shows that adaptive modulation gives optimum results for given SNR. (Refer fig. 4)

The plot (fig. 5) has been shown for dynamic MIMO channel model (with fading) and MU-MIMO that is two transmit and receive antennas which shows that with the increasing SNR values the data rate increases with increasing error for higher modulation schemes. Here due to fading and multi transmit and receive antenna error increases for all modulation schemes to larger values for given SNR. The statistical values as well as plot of error shows that adaptive modulation gives optimum results for given SNR. (Refer fig. 5) 
Volume 6 Issue 6 June, 2018

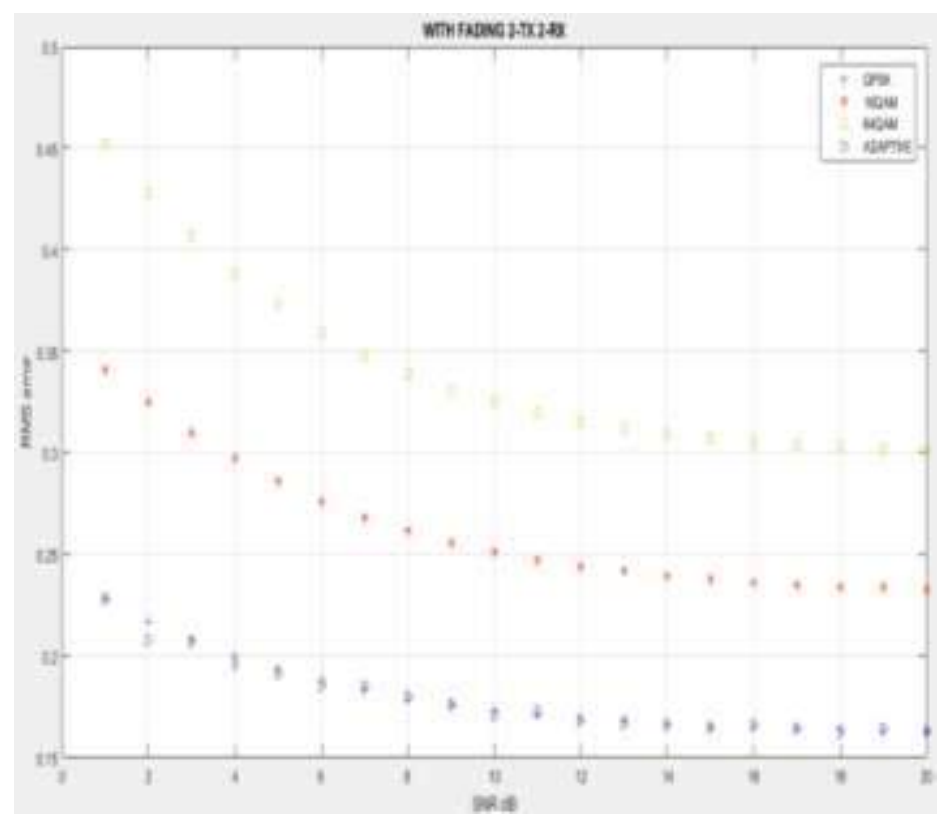

Fig 5: Plot of RMS error vs SNR for with fading 2Tx 2-Rx

\section{FUTURE SCOPE}

The work done here is achieved at constant value of precoder matrix, we can also include PMI, and RI Feedback in study and then calculate performance of MIMO transreceiver in LTE.

\section{REFERENCES}

[1]. Yamindi J-B, Hong J and Wu M-Q 2012 “The optimization capacity of the MU-MIMO with channel quality information", IEEE 16th International Symposium on Consumer Electronics (ISCE), pp 1-5, doi: 10.1109/ISCE.2012.6241685.

[2]. Roshni Sahu, Sagar Kumar Sen, Sahas Patil “A Simulation Design of LTE Communication System under Adaptive Modulation Schemes", Volume 7 Issue No.6, 2017.

[3]. Houman Zarrinkoub PhD "Modeling a 4GLTE System in MATLAB”, 2012. The Math Works, Inc., 2012. BalaALhajiSalihu, YangDacheng, ZhangXin, SuleimanZubair "Evolution of Physical Downlink Control
Channel (PDCCH) for LTE-Advanced system". University technology Malaysia2014.

[4]. Gessese Mengistu Kebede and Oladele Olayinka Paul "Performance Evaluation of LTE Downlink with MIMO Techniques", Thesis no: MEE10:104 November, 2010.

[5]. S.S.Hadi and T.C.Tiong "Adaptive Modulation and Coding for LTE Wireless Communication" Department of Electrical and Computer Engineering, Faculty of Engineering and Science, Curtin University, Miri, Sarawak, Malaysia 2016.

[6]. Ramkumar Gowrishankar, Mehmet Fatih Demrikol and Marc Fossorier "Adaptive Modulation Based MIMO System", December 2005.

[7]. Mohamed Lassaad Ammari and Francois Gagnon "Adaptive modulation for DFE equalized systems", Volume 13, 2014.

[8]. Catreux S, Gesbert D and Heath R W June 2002 "Adaptive modulation and MIMO coding for broadband wireless data networks" IEEE Commun. Mag. vol $40 \mathrm{pp}$ 108-115.

[9]. Goldsmith A J and Chua S-G 1999 "Adaptive Modulation and coding for fading channels" Proc. IEEE Information Theory and Communications Workshop pp 24-26 doi: 10.1109/ITCOM. 1999.781396.

[10]. Sharma A and De S November 2011 "Exploiting fading dynamics along with amc for energy- efficient transmission over fading Channels" IEEE Communications Letters 15 pp 1218-20 doi:0.19/LCOMM.2011.090911.111472. 